\title{
On the hydrogen neutral outflowing disks of $B[e]$ supergiants
}

\author{
M. Kraus ${ }^{1}$, M. Borges Fernandes ${ }^{2}$, and F. X. de Araújo ${ }^{3}$ \\ 1 Astronomical Institute AV ČR, Fričova 298, 25165 Ondřejov, Czech Republic \\ e-mail: kraus@sunstel.asu.cas.cz \\ 2 Observatório do Valongo (UFRJ), Ladeira do Pedro Antonio 43, Rio de Janeiro 20080-090, Brazil \\ e-mail: borges@ov.ufrj.br \\ 3 Observatório Nacional, Rua General José Cristino 77, 20921-400 São Cristovão, Rio de Janeiro, Brazil \\ e-mail: araujo@on.br
}

Received 31 August 2006 / Accepted 19 November 2006

\section{ABSTRACT}

\begin{abstract}
Context. $\mathrm{B}[\mathrm{e}]$ supergiants are known to possess geometrically thick dusty disks. Disk-forming wind models in the literature have, however, been found to be insufficient in reproducing the observed dust emission. This problem arises due to the severe assumption that, as for classical Be stars, the near-infrared excess emission originates in the disk. Modeling of the free-free and free-bound emission therefore results in an upper limit for the disk mass loss rate as well as for the disk opacity. Dust condensation in the disk can thus severely be hampered.

Aims. In order to overcome the dust formation problem, and based on our high-resolution optical spectroscopy and model results, we propose a revised scenario for the non-spherical winds of B[e] supergiants: a normal B-type line-driven polar wind and an outflowing disk-forming wind that is neutral in hydrogen at, or very close to the stellar surface.

Methods. We concentrate on the pole-on seen LMC B[e] supergiant R 126 and calculate the line luminosities of the optical [OI] emission lines and their emergent line profiles with an outflowing disk scenario. In addition, we compute the free-free and free-bound emission from a line-driven polar wind and model the spectral energy distribution in the optical and near-infrared.

Results. Good fits to the [OI] line luminosities are achieved for an outflowing disk that is neutral in hydrogen right from the stellar surface. Neutral thereby means that hydrogen is ionized by less than $0.1 \%$. Consequently, the free-free and free-bound emission cannot (dominantly) arise from the disk and cannot limit the disk mass loss rate. The hydrogen neutral outflowing disk scenario therefore provides an ideal environment for efficient dust formation. The spectral energy distribution in the optical and near-infrared range can be well fitted with the stellar continuum plus free-free and free-bound emission from the polar line-driven wind. Our modeling further delivers minimum values for $\dot{M}_{\text {disk }} \gtrsim 2.5 \times 10^{-5} M_{\odot} \mathrm{yr}^{-1}$ and for the density contrast between equatorial and polar wind of $\sim 10$.
\end{abstract}

Key words. stars: mass-loss - stars: winds, outflows - supergiants - stars: individual: R 126 - stars: emission-line, Be stars: early-type

\section{Introduction}

The spectra of $\mathrm{B}[\mathrm{e}]$ supergiants show the so-called hybrid character which is defined by the co-existence of a line-driven polar wind and a high density but low velocity equatorial wind (Zickgraf et al. 1985). The latter is assumed to form a disk-like structure. This disk is the location of the low-ionized metals, of the sometimes observed $\mathrm{CO}$ and $\mathrm{TiO}$ molecular emission bands (McGregor et al. 1988, 1989; Zickgraf et al. 1989) as well as of the hot dust, pronounced in the mid-IR excess emission (e.g. Zickgraf 1992). The disk formation mechanism, as well as the disk structure, are however still unsolved problems.

Porter (2003) has studied the possible nature of these disks by modeling their spectral energy distribution (SED). He found that neither an outflowing disk-forming wind nor a Keplerian viscous disk in their simplest form can easily account for the observed free-free and dust emission self-consistently.

The optical spectra of $\mathrm{B}[\mathrm{e}]$ supergiants exhibit strong [OI] emission (see e.g. Kraus \& Borges Fernandes 2005; Kraus et al. 2006; and Fig. 1). Besides other typical features like strong Balmer emission, FeII and [FeII] emission, and mid-IR excess emission, the [OI] emission lines are one of the main characteristics of stars with the B[e] phenomenon (Lamers et al. 1998). The fact that OI and HI have about equal ionization potentials requests that the [OI] emission region must be neutral in hydrogen. Its most plausible location is therefore the high-density disk.

Test calculations for an outflowing disk scenario, that were aimed to reproduce the observed strong [OI] line luminosities, have been performed for some Magellanic Cloud B[e] supergiants (Kraus \& Borges Fernandes 2005; Kraus et al. 2006). These computations revealed that, in order to keep the disk mass loss rates at reasonable values, the disk material must be neutral in hydrogen either at or at least very close to the stellar surface, resulting in a hydrogen neutral disk.

In this paper we present model results for the Large Magellanic Cloud (LMC) B[e] supergiant R 126. We use a simple outflowing disk scenario and request that the disk is neutral in hydrogen right from the stellar surface. We show that with such a model we can explain observations like the strong [OI] line luminosities. In addition, this scenario provides a way out of the dust formation problem mentioned by Porter (2003). The paper is structured as follows: in Sect. 2 we give details on the test star and on our observations. Section 3 introduces the problem of the nature of the $\mathrm{B}[\mathrm{e}]$ supergiants disks. We emphasize the need of a revised disk model and propose the hydrogen neutral disk as a reasonable scenario. In Sect. 4 we present our hydrogen neutral disk model and calculate the emerging [OI] line luminosities 


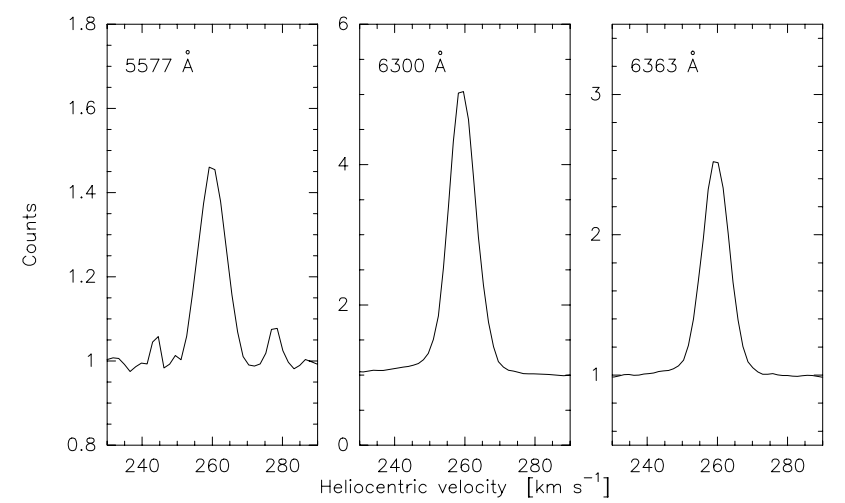

Fig. 1. The [OI] lines in the FEROS spectrum of R 126. They all show a redshift of about $260 \mathrm{~km} \mathrm{~s}^{-1}$ caused by the radial velocity of the LMC.

as well as their line profiles. In Sect. 5 we fit the SED in the optical and near-IR range by calculating the free-free and freebound emission from the polar wind. In addition, we determine the density ratio between equatorial and polar wind components and show that our model results in a lower limit. The validity of our assumptions is discussed in Sect. 6, and the conclusions are summarized in Sect. 7.

\section{The $L M C B[e]$ supergiant $R 126$}

The LMC B[e] supergiant R 126 (HD 37974, LHA 120-S 127) is supposed to have a pole-on seen disk. This orientation has the following advantages (see Fig. 2): (i) it guarantees that we see all of the [OI] emission and none is hidden by the star or absorbed by the dusty disk because the [OI] emission originates from regions close to the star where no dust has yet formed, and (ii) it simplifies the analysis due to the high degree of symmetry. R 126 has also been studied by Porter (2003) so that we can directly compare our results. The stellar parameters of this object have been derived by Zickgraf et al. (1985) who found: $T_{\text {eff }}=22500 \mathrm{~K}, L_{*}=1.2 \times 10^{6} L_{\odot}, R_{*}=72 R_{\odot}$, and an interstellar extinction value of $E(B-V)=0.25$. These values will be used for our calculations throughout the paper.

We obtained high- and low-resolution optical spectra of $\mathrm{R} 126$ at the ESO 1.52-m telescope (agreement ESO/ON-MCT) in La Silla (Chile) using the Fiber-fed Extended Range Optical Spectrograph (FEROS) and the Boller \& Chivens spectrograph, respectively. FEROS is a bench-mounted Echelle spectrograph with fibers, that cover a sky area of 2 arcsec, located at the Cassegrain focus with a wavelength coverage from $3600 \AA$ to $9200 \AA$ and a spectral resolution of $R=55000$ (in the region around $6000 \AA$ ). It has a complete automatic on-line reduction, which we adopted. The spectra were taken on December 18, 1999 , with an exposure time of $4500 \mathrm{~s}$. The $S / N$ ratio in the $5500 \AA$ A region is approximately 100 .

The low-resolution Boller \& Chivens (B\&C) spectrum was taken on October 31, 1999, with an exposure time of $900 \mathrm{~s}$ and a slit width of 4 arcsec. The instrumental setup employed provides a resolution of $\sim 4.6 \AA$ in the range of $3800-8700 \AA$. In the $5500 \AA$ continuum region, the $S / N$ ratio is aproximately 200 . The B\&C spectrum was reduced using standard IRAF tasks, such as bias subtraction, flat-field normalization, and wavelength calibration. We have done absolute flux calibration using spectrophotometric standards from Hamuy et al. (1994).

Equivalent widths and line intensities in both linearized spectra have been measured using the IRAF task that computes

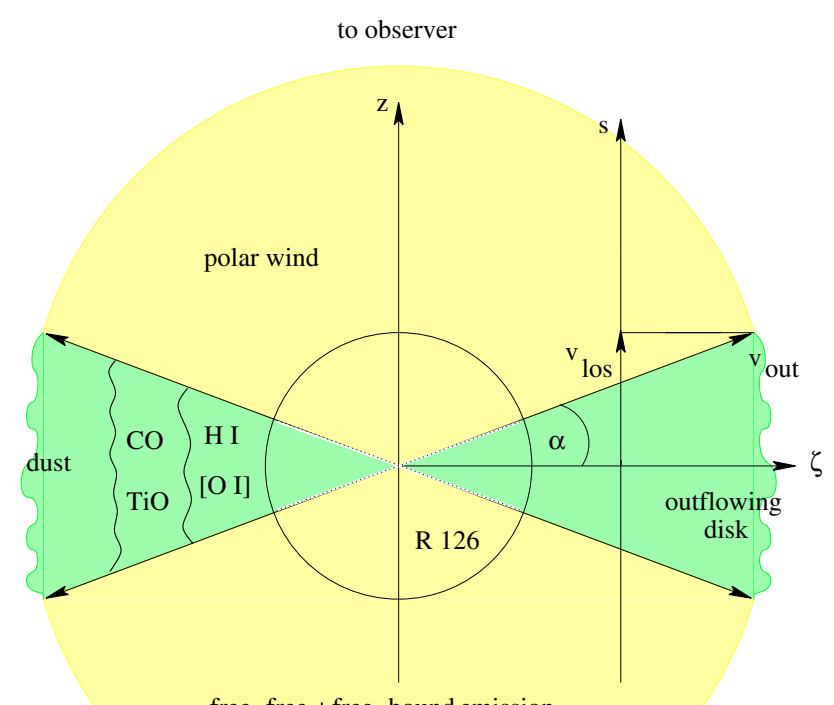

free-free + free-bound emission

Fig. 2. Sketch of the pole-on seen disk around R 126 (not to scale). The disk (dark grey shaded) is assumed to be neutral in hydrogen right from the stellar surface, giving raise to [OI] emission. Further out, molecules and dust will form. The disk material moves outwards with $v_{\text {out }}$, resulting in an observable line-of-sight velocity $\left(v_{\mathrm{los}}\right)$. Free-free and freebound emission is restricted to the (polar) wind (light grey shaded). The system is axially symmetric (around $z$-axis) and symmetric with respect to the disk mid-plane.

the line area above the adopted continuum. Uncertainties in our measurements come mainly from the position of the underlying continuum and we estimate the errors to be about $20 \%$. The emerging [OI] lines resolved within the FEROS spectrum are shown in Fig. 1.

\section{On the nature of $B[e]$ supergiant stars' disks}

The nature of the $\mathrm{B}[\mathrm{e}]$ supergiant stars' disks is a long-standing problem. Recently, Porter (2003) investigated the possibility of dust formation in the disk of the $\mathrm{B}[\mathrm{e}]$ supergiant R 126 for two different approaches: an outflowing disk-forming wind, and a Keplerian viscous disk. He found that both models failed in reproducing the observed dust and free-free emission selfconsistently. And he suggested a way out of this by allowing for substantial alteration especially of the exponent of the radial density distribution from $\rho \sim r^{-2}$ to a considerably flatter one of $r^{-1.7}$. The advantage of a flatter density profile is that at every location in the disk a higher density (and hence opacity) is maintained, allowing for more efficient dust condensation and therefore for an enhanced dust emission over the disk. Such a modification finally resulted in a good fit to the SED of R 126 (see Fig. 6 of Porter 2003), but for the price that the disk density distribution now no longer corresponds to a constant velocity outflow (which requests $\rho \sim r^{-2}$ ). A possible interpretation of such a flatter density profile might be that the disk slows down with distance, a scenario that lacks any observational evidence.

To test Porter's modified outflowing disk scenario, we took the parameters of his best-fit model and calculated the emerging [OI] line luminosities. These turned out to be at least a factor of 50 lower than the values we observed. This means that we need 
a much higher density within the [OI] line forming region than can be provided by Porter's disk model.

The most severe limitation in his model calculations was the assumption that, as in the case of classical Be stars, the freefree emission arises in the high density disk, while contributions from the polar wind are negligible. Fitting the near-IR part of the SED with free-free emission from the outflowing disk therefore determines the disk mass loss rate. Consequently, this value is an upper limit for the disk density and hence opacity. Dust can only condensate at distances with reasonable values of temperature and opacity. An upper limit for the disk density, defined by the free-free emission, can therefore severely hamper efficient dust formation in the disk, which turned out to be the major reason why Porter (2003) could not fit the observed dust emission of R 126 with the original outflowing disk scenario.

The detection of strong [OI] line emission in the spectra of e.g. $\mathrm{B}[\mathrm{e}]$ supergiants requests that there must be a rather high density disk region which is neutral in hydrogen $(T \lesssim 10000 \mathrm{~K})$, but still hot enough $(6000 \lesssim T[\mathrm{~K}] \lesssim 8000)$ for effective excitation of the levels in OI. First test calculations that were aimed to reproduce the [OI] line luminosities with an outflowing disk scenario have been performed by Kraus \& Borges Fernandes (2005) and Kraus et al. (2006). These computations showed that, in order to keep the disk mass loss rates at reasonable values, the disks must be neutral in hydrogen either at, or at least very close to the stellar surface.

The existence of hydrogen neutral disks around $\mathrm{B}[\mathrm{e}]$ supergiants is additionally supported by recent ionization structure calculations in non-spherically symmetric winds. These computations have shown that the wind material in the equatorial plane can indeed recombine at, or close to the stellar surface if either the equatorial mass flux is enhanced compared to the polar one (Kraus \& Lamers 2003), or even decreased in combination with a reduction in surface temperature due to rapid rotation of the star (Kraus 2006; Kraus \& Lamers 2006).

Having a hydrogen neutral disk around a luminous object is therefore not as unrealistic as it might seem at first glance. In fact, it has several striking advantages: if the disk is predominantly neutral, the free-free and free-bound emission can no longer be generated in the disk, but must (mainly) originate within the polar wind. The disk mass loss rate is therefore not strictly linked to the free-free emission causing the near-IR excess. Hence, the disk mass loss rate might be (much) higher than the upper limit used by Porter (2003). A higher disk density is certainly needed to reproduce the observed [OI] line luminosities. And finally, a higher disk density will increase the disk opacity and will allow for more efficient dust condensation in the disk, needed to reproduce the observed IR excess.

In the following we will investigate and test the suggested model scenario of a hydrogen neutral disk around a luminous object by analysing in detail the observed [OI] lines from the $\mathrm{B}[\mathrm{e}]$ supergiant R 126 .

\section{The (hydrogen) neutral disk}

\subsection{The disk geometry}

The disk is assumed to be wedge-shaped (see sketch in Fig. 2) and we use the same value of $\alpha=10^{\circ}$ for the constant disk half opening angle as Porter (2003). For simplicity we assume that the disk has a constant outflow velocity within the [OI] emitting region. The influence of a velocity distribution on the final results is discussed in Sect. 6.

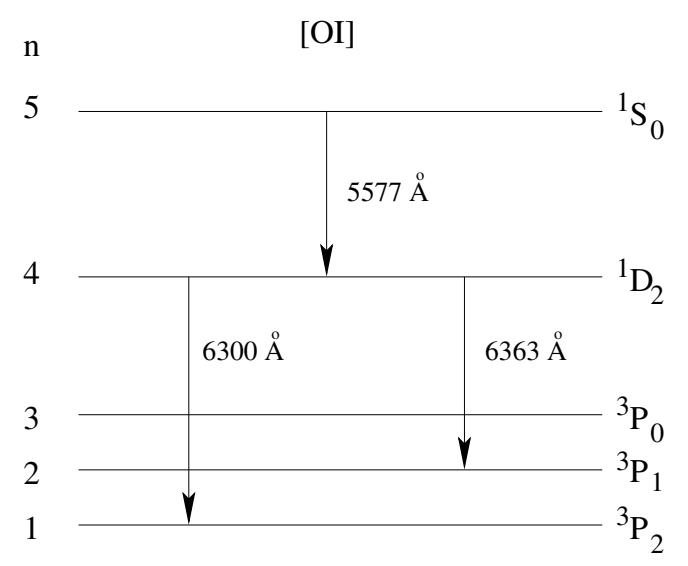

Fig. 3. Sketch of the five lowest energy levels within OI from which the forbidden lines arise. The transitions of our interest are indicated.

In a disk-forming wind of constant outflow velocity, the hydrogen particle density distribution at all latitudes within the disk can be written in the form

$n_{\mathrm{H}}(r)=n_{\mathrm{H}}\left(R_{\mathrm{in}}\right) \frac{R_{\text {in }}^{2}}{r^{2}}$.

The parameter $n_{\mathrm{H}}\left(R_{\mathrm{in}}\right)$, given by

$n_{\mathrm{H}}\left(R_{\text {in }}\right)=\frac{1}{\mu m_{\mathrm{H}}}\left(\frac{R_{*}}{R_{\text {in }}}\right)^{2} \frac{F_{\mathrm{m}, \mathrm{disk}}}{v_{\text {out }}}$

with the disk mass flux, $F_{\text {m,disk }}$, and the (constant) disk outflow velocity, $v_{\text {out }}$ (see Fig. 2), describes the density at the inner radius, $R_{\text {in }}$, of the [OI] emitting region. This inner radius corresponds to the hydrogen recombination distance in the disk and is in principle a free parameter in our calculations, but here we use $R_{\text {in }}=R_{*}$ since this defines a lower limit to the disk density needed to reproduce the [OI] line luminosities. The validity of our assumption that the disk is neutral in hydrogen right from the stellar surface is discussed in Sect. 6. The oxygen and electron density distributions are parameterized in terms of the hydrogen density, i.e.

$n_{\mathrm{O}}(r)=q_{\mathrm{O}} n_{\mathrm{H}}(r) \quad$ and $\quad n_{\mathrm{e}}(r)=q_{\mathrm{e}} n_{\mathrm{H}}(r)$.

The parameters $q_{\mathrm{O}}$ and $q_{\mathrm{e}}$ describe the relative abundances of oxygen and electrons with respect to hydrogen. For $q_{0}$ we use a typical LMC abundance value of $1 / 3$ solar, with a solar oxygen abundance of $q_{\mathrm{O} \text {,solar }}=6.76 \times 10^{-4}$ (Grevesse \& Sauval 1998). Since we do not know the ionization fraction of the disk a priori, we keep $q_{\mathrm{e}}$ as a free parameter.

\subsection{The $\left[\mathrm{O}_{I}\right]$ lines and the disk ionization}

The forbidden emission lines arise from the five lowest energy levels of the OI atom that are excited via collisions with free electrons. These levels are shown schematically in Fig. 3. For simplicity, we labeled them from 1 to 5 corresponding to their increasing energy. The three lines resolved in our high-resolution optical spectra are indicated; they belong to the transitions $5 \longrightarrow$ $4(5577 \AA), 4 \longrightarrow 1(6300 \AA)$, and $4 \longrightarrow 2(6363 \AA)$.

While the lines $6300 \AA$ and $6363 \AA$ arise from the same upper level, which means that their line ratio is independent of the density and temperature but is determined purely by atomic parameters, the situation is different for line ratios with the $5577 \AA$ line. This line arises from the highest energy level in our 
5-level atom (Fig. 3). It is therefore obvious that the $5577 \AA$ line will only become strong if level 5 is populated efficiently, either due to a huge number of available free electrons or by electrons with rather high energy. Line ratios with the $5577 \AA$ line are thus sensitive tracers for the temperature and ionization fraction in the disk. In our spectra, the $6300 \AA$ line is the one with highest luminosity. We will therefore use the $6300 \AA / 5577 \AA$ line luminosity ratio to investigate density and temperature in the disk around R 126.

Forbidden lines are optically thin. The emissivity at any location $r$ in the disk of a transition $n \rightarrow m$ is given by $j_{n, m}(r)=$ $h v_{n, m} n_{n}(r) A_{n, m}$ where $n_{n}(r)$ is the level population of the upper level, $\mathrm{n}$, at this location. The level populations are calculated by solving the statistical equilibrium equations in our 5-level atom. Collision parameters are taken from Mendoza (1983) and atomic parameters from Wiese et al. (1966) and from Kafatos \& Lynch (1980). The line luminosity finally follows from integration of the emissivity over the emitting disk volume.

Due to the nearly equal ionization potentials of $\mathrm{O}$ and $\mathrm{H}$, the [OI] emission must arise in those parts of the disk in which hydrogen is predominantly neutral. This does not mean that hydrogen must be $100 \%$ neutral, since effects like collisional ionization that might take place in the high density disk could keep the hydrogen material ionized at a (very) low level, providing free electrons to collisionally excite OI. Further sources of free electrons in the disk are elements like $\mathrm{Mg}, \mathrm{Si}, \mathrm{Fe}, \mathrm{Al}$, etc. with ionization potentials (much) lower than $13.6 \mathrm{eV}$. These metals will remain ionized at a certain fraction even in a completely hydrogen neutral environment. The ionization fraction, $q_{\mathrm{e}}$, in the disk is therefore the sum of all free electrons provided by hydrogen and the metals, i.e. $q_{\mathrm{e}}=q_{\mathrm{H}^{+}}+q_{\text {Metals }^{+}}$.

To get a handle on the possible individual contributions to the total number of free electrons in the disk, we make a first guess. If all possible elements with ionization potential lower than $13.6 \mathrm{eV}$ would be fully singly ionized (including also $\mathrm{C}$ with $\chi=11.26 \mathrm{eV}$ and $\mathrm{Cl}$ with $\chi=12.97 \mathrm{eV}$ ), then a maximum number of free electrons of $q_{\text {Metals }}{ }^{+} \leq 1.57 \times 10^{-4}$ would be provided by the metals in the disk. This number accounts for an LMC metallicity of $1 / 3$ solar. On the other hand, if only $1 \%$ of hydrogen in the disk remains ionized, the ionization fraction would be $q_{\mathrm{H}^{+}}=10^{-2}$ which is at least a factor 100 higher than what can be expected from the metals. Our assumption of a hydrogen neutral disk around R 126 therefore requests that the total disk ionization fraction should be $q_{\mathrm{e}}<10^{-2}$.

To derive the ionization fraction in the disk of $\mathrm{R} 126$ we calculated the line luminosities and the $6300 \AA / 5577 \AA$ line ratio for a large range of $q_{\mathrm{e}}$ values and for different temperatures. These calculations were done in several steps: for a given ionization fraction and temperature we first varied the density parameter, $F_{\text {m,disk }} / v_{\text {out }}$ (see Eq. (2)), in order to reproduce the observed line luminosity of the $6300 \AA$ line. This density parameter is plotted as a function of $q_{\mathrm{e}}$ in the lower panel of Fig. 4. With the proper density parameter, we then calculated the line luminosities of all [OI] lines and derived the $6300 \AA / 5577 \AA$ line ratio. This line ratio is plotted as a function of $q_{\mathrm{e}}$ in the upper panel of Fig. 4. In this plot we also included the observed line ratio, shown as the dotted line.

Our calculations cover a temperature range between $7500 \mathrm{~K}$ and $9000 \mathrm{~K}$. The curves in the upper panel of Fig. 4 all show the same trend of a decrease in line ratio with increasing ionization fraction. This effect is clear, because the number of free electrons determines the collisional excitation rate of the levels. The more electrons, the higher the probability for collisional

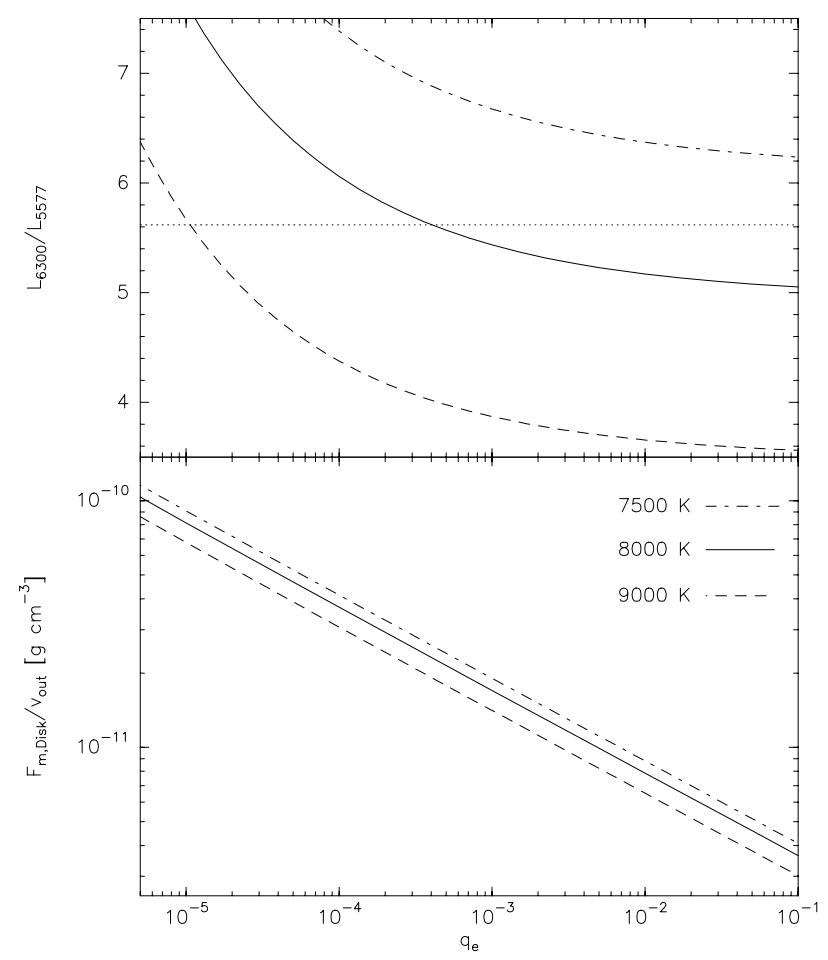

Fig. 4. Upper panel: variation of the line ratio with disk ionization fraction. The three curves are for the different temperatures as indicated. The dotted line represents the observed line ratio from $\mathrm{R} 126$. It indicates a disk temperature in the range $9000 \mathrm{~K}$ to about $8000 \mathrm{~K}$ with corresponding ionization fractions of $10^{-5}$ and $4 \times 10^{-4}$, respectively. Lower panel: density parameter of the disk found from fitting the observed $6300 \AA$ line luminosity over a huge range of ionization fractions. For details see text.

excitation even of the level with highest energy (i.e. level 5) from which the $5577 \AA$ line arises. The second obvious effect is the decrease in line ratio with increasing temperature, since the temperature determines the kinetic energy of the free electrons. For low temperatures, the energy of the free electrons is not high enough to effectively excite level 5 , reducing the $5577 \AA$ line emission and hence increasing the line ratio.

For the three temperature values used to calculate the theoretical curves in Fig. 4 we find agreement with the observed line ratio for the following parameter combinations: for $9000 \mathrm{~K}$ we find an ionization fraction of $10^{-5}$, and for $8000 \mathrm{~K}$ we find an ionization fraction of $4 \times 10^{-4}$. For a temperature of $7500 \mathrm{~K}$, we cannot reproduce the observed line ratio.

Our calculations show that when accounting for the line luminosity and line ratio observed from $\mathrm{R} 126$, the ionization fraction is rather low for high temperatures, while an ionization fraction of up to $10 \%$ might exist for a temperature as low as $7800 \mathrm{~K}$. This behaviour is only strictly valid for a given observed line luminosity. In order to restrict the range of valid or reasonable disk parameters, we take account of the fact that the ionization fraction can only increase with increasing temperature, since collisional ionization will act more efficiently. This indicates that for the disk around R 126 we can exclude high temperatures $(\gtrsim 9000 \mathrm{~K})$ as well as low temperatures ( $\lesssim 8000 \mathrm{~K})$, and we conclude that the [OI] line forming region in the disk of $\mathrm{R} 126$ has a temperature range of $\sim 8000 \ldots 8500 \mathrm{~K}$ with a corresponding ionization fraction range of $5 \times 10^{-4} \gtrsim q_{\mathrm{e}} \gtrsim 5 \times 10^{-5}$. This range in ionization fraction states that less than $0.05 \%$ of 


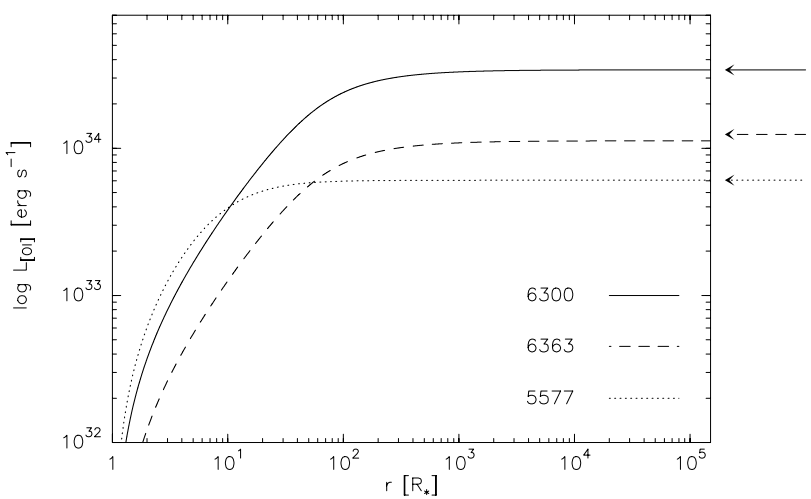

Fig. 5. Calculated increase in [OI] line luminosities from the hydrogen neutral disk around R 126 . The observed, extinction corrected values are indicated with arrows. The observations have been taken with an aperture radius of $2^{\prime \prime}$, corresponding to an outer edge of the observed wind of about $1.5 \times 10^{5} R_{\odot}$ at the distance of the LMC. This value is used as the extent of the line luminosity integration, but the [OI] line luminosities clearly saturate already within about $500 R_{*}$.

hydrogen in the disk is ionized. We can therefore conclude that the disk around R 126 is indeed neutral in hydrogen.

Figure 5 shows the increase in [OI] line luminosities calculated for a disk of $8000 \mathrm{~K}$ and an ionization fraction of $4 \times 10^{-4}$. The lines saturate within a few hundred stellar radii. The observed values are indicated by the arrows to the right.

\subsection{Disk outflow velocity and mass loss rate}

For a temperature of $8000 \mathrm{~K}$ as derived in the previous section, we end up with a density parameter of $F_{\mathrm{m} \text {,disk }} / v_{\text {out }} \simeq 2.2 \times$ $10^{-11} \mathrm{~g} \mathrm{~cm}^{-3}$ for the disk of R 126 (see Fig. 4). To compare our results with those of Porter (2003), we need to convert this density parameter into a mass loss rate. For this, the outflow velocity, $v_{\text {out }}$, of the disk is needed. For a pole-on seen disk with radially outflowing material, the maximum observable line-of-sight velocity, $v_{\text {los }}$, is linked to the outflow velocity via $v_{\text {los }}=v_{\text {out }} \sin \alpha$ (see Fig. 2). The range in possible $v_{\text {los }}$ values over the different disk outflow directions above and below the disk mid-plane leads to symmetrical doppler shifts of the line center, while thermal and turbulent velocities as well as the spectral resolution of the instrument result in a Gaussian profile of the line. Thus, optically thin lines formed in a pole-on seen outflowing disk should be symmetric with respect to their laboratory wavelength. The observed [OI] lines are indeed symmetric (Fig. 1) and their profiles show a Gaussian shape with a $F W H M$ of $\sim 9.2 \mathrm{~km} \mathrm{~s}^{-1}$.

To derive a possible outflow contribution from the observed lines, we first calculate the following test profiles: (i) a pure Gaussian line profile and (ii) a pure outflowing disk profile. The results are shown in the left panel of Fig. 6. The calculated lines are normalized to their maximum value, and $v_{\text {gauss }}$ and $v_{\text {out }}$ are chosen such that they result in a $F W H M$ value of $9.2 \mathrm{~km} \mathrm{~s}^{-1}$. The pure outflowing disk (neglecting the influence of FEROS' spectral resolution) results in a flat-topped profile with steeply rising wings. The narrow observed [OI] lines show a better agreement with a Gaussian line shape, and the fact that the observed [OI] lines have widths that are only marginally broader than the velocity resolution of FEROS indicates that the intrinsic FWHM of the lines can only be on the order of $2-3 \mathrm{~km} \mathrm{~s}^{-1}$. Such a low intrinsic $F W H M$ can be ascribed to pure thermal broadening.

Since spectra taken with FEROS are not flux-calibrated, the reproduction of the line profiles can only give qualitative results.

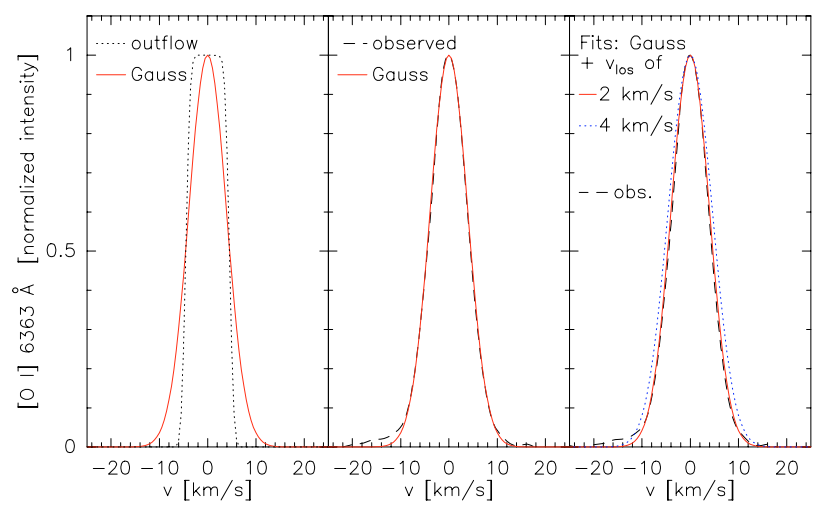

Fig. 6. Normalized profiles of the [OI] $6363 \AA$ line. Left: theoretical profiles for either a pure Gaussian or a pure outflowing disk profile. Middle: fit of a Gaussian profile with $F W H M$ of $9.2 \mathrm{~km} \mathrm{~s}^{-1}$ resulting from FEROS' spectral resolution plus thermal motion of the gas. Right: fit of the same Gaussian including an outflow component causing $v_{\text {los }}$ of either $2 \mathrm{~km} \mathrm{~s}^{-1}$ or $4 \mathrm{~km} \mathrm{~s}^{-1}$. While the first one still gives a reasonable fit, the latter one clearly results in a too broad line profile.

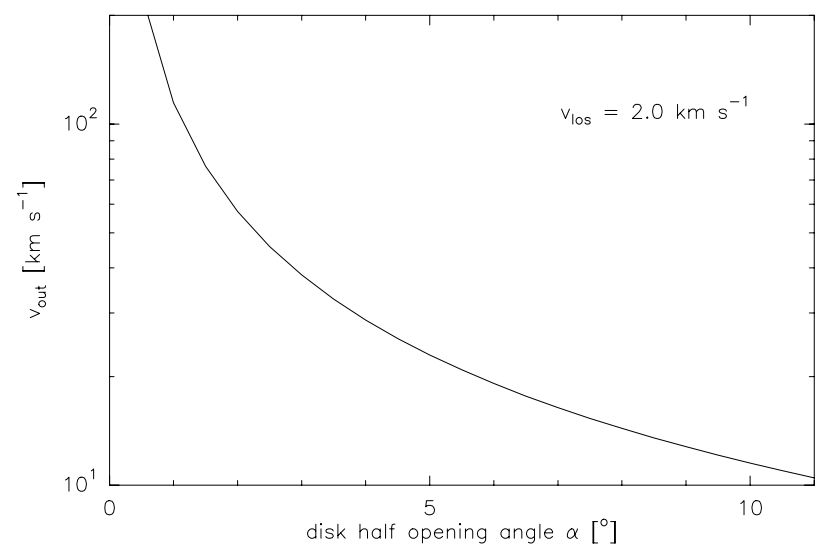

Fig. 7. Disk outflow velocity as function of disk half opening angle for $v_{\text {los }}=2 \mathrm{~km} \mathrm{~s}^{-1}$ as derived from the [OI] line profile fitting.

We took exemplarily the line profile of the $6363 \AA$ line obtained with FEROS, corrected it for the LMC radial velocity component of $260 \mathrm{~km} \mathrm{~s}^{-1}$, and normalized the line to its maximum intensity. This normalized line profile is what we intend to fit.

We first used a pure Gaussian line profile with $F W H M$ of $9.2 \mathrm{~km} \mathrm{~s}^{-1}$ (resulting from FEROS' spectral resolution plus thermal broadening of the line) which results in a good fit (mid panel of Fig. 6).

Next we combined this Gaussian profile with an outflowing disk profile for different values of $v_{\text {los }}$. This was done to see for which value of $v_{\text {los }}$ the line profile starts to alter. We found that a possible $v_{\text {los }}$ contribution must be smaller than $\sim 2 \mathrm{~km} \mathrm{~s}^{-1}$ (right panel of Fig. 6) resulting in an outflow velocity of $v_{\text {out }} \lesssim 11.5 \mathrm{~km} \mathrm{~s}^{-1}$ for $\alpha=10^{\circ}$. The value of $\alpha$ should be taken as an upper limit since the range of possible $\mathrm{B}[\mathrm{e}] \mathrm{su}-$ pergiant disk values has been estimated by Zickgraf (1992) to be $\alpha=5-10^{\circ}$. A thinner disk will result in a higher outflow velocity for the same value of $v_{\text {los }}$ (see Fig. 7). Nevertheless, even considering a thinner disk, the outflow velocity is considerably smaller than the values of $60-80 \mathrm{~km} \mathrm{~s}^{-1}$ usually ascribed to the disks of $\mathrm{B}[\mathrm{e}]$ supergiants. In the case of R 126, no good estimates for the real disk outflow velocity exist and only a detailed investigation of the line profiles from emission lines that might be connected with the disk will help to clarify this point. 
An outflow velocity of $11.5 \mathrm{~km} \mathrm{~s}^{-1}$ as derived above together with the disk density parameter derived from the [OI] line luminosity calculations and a disk opening angle of $20^{\circ}$ results in a total disk mass loss rate of $\dot{M}_{\text {disk }} \simeq 2.5 \times 10^{-5} M_{\odot} \mathrm{yr}^{-1}$. This is about a factor of 10 higher than the value used by $\operatorname{Porter}^{1}$ (2003), and well in agreement with the postulated need for a higher disk density in order to account for the observed [OI] line luminosities of R 126.

Based on recent observations with the Spitzer Space Telescope Infrared Spectrograph, Kastner et al. (2006) derived a total dust mass of the disk around R 126 of $\sim 3 \times 10^{-3} M_{\odot}$, and they claimed that the dust is contained between $120 \mathrm{AU}$ and $2500 \mathrm{AU}$. In order to compare this dust mass with our derived disk mass loss rate, we assume a gas to dust ratio of about 100 and convert the total mass into a disk mass loss rate for our outflowing disk model. We end up with a density parameter of $F_{\text {m,disk }} / v_{\text {out }} \simeq 3 \times 10^{-10} \mathrm{~g} \mathrm{~cm}^{-3}$ and a disk mass loss rate of $\dot{M}_{\text {disk }} \simeq 3.4 \times 10^{-4} M_{\odot} \mathrm{yr}^{-1}$. This is about 170 times higher than the value used by Porter (2003) and about 13 times higher than our value, and confirms that the disk around R 126 must indeed be more massive than previously thought.

In addition, the inner edge of the dusty disk of $120 \mathrm{AU}$, which corresponds to about $360 R_{*}$, lies within our [OI] saturation region. This confirms that our value for the disk mass loss rate is indeed a lower limit.

\section{The polar wind}

With our assumption of a hydrogen neutral disk right from the stellar surface, the free-free and free-bound emission is restricted to the ionized wind parts (see Fig. 2). At all latitudes above and below the equatorial disk we assume that the star has a normal B-type line-driven wind, which we refer to as the polar wind. Its radial density distribution, following from the equation of mass continuity, is

$n_{\mathrm{H}}(r)=\frac{1}{\mu m_{\mathrm{H}}}\left(\frac{R_{*}}{r}\right)^{2} \frac{F_{\mathrm{m}, \mathrm{pol}}}{v(r)}$,

where $F_{\mathrm{m} \text {,pol }}$ is the mass flux of the polar wind. The velocity increase in line-driven winds can be approximated with a $\beta$-law with $\beta$ typically in the range of $0.8-1.0$ for hot star winds (see e.g. Lamers \& Cassinelli 1999). We assume that the wind temperature is constant which allows for a simplified treatment of the intensity calculation. At every point along the impact parameter, $\zeta$ (see Fig. 2), the intensity is given by

$I_{v}=B_{v}(T)\left(1-\mathrm{e}^{-\tau(\zeta)}\right)$.

The optical depth $\tau_{v}(\zeta)=\int \kappa_{v}(\zeta, s) \mathrm{d} s$ is defined by the lineof-sight integral (with the line-of-sight perpendicular to the disk mid-plane) over the absorption coefficient, $\kappa_{v}$, defined by

$\kappa_{\nu}(\zeta, s) \simeq 3.692 \times 10^{8} \frac{n_{\mathrm{e}}^{2}(\zeta, s)}{v^{3} T^{1 / 2}}\left(1-\mathrm{e}^{-\frac{h \nu}{k T}}\right)\left(g_{\mathrm{ff}}+g_{\mathrm{fb}}\right)$,

and integration limits depending on the geometry of the system (Fig. 2). The parameters $g_{\mathrm{ff}}$ and $g_{\mathrm{fb}}$ are the gaunt factors for freefree and free-bound processes, respectively, and have been calculated (e.g. by Kraus 2000) over a large frequency range and for

${ }^{1}$ Note that the parameter $\dot{M}_{\mathrm{d}}$ used by Porter does not describe the total disk mass loss rate. Instead, his value of $\dot{M}_{\mathrm{d}}=10^{-5} M_{\odot} \mathrm{yr}^{-1}$ needs to be multiplied by the solid angle of the disk and divided by $4 \pi$. The total disk mass loss rate in his models is therefore $\dot{M}_{\text {disk }} \simeq 2 \times 10^{-6} M_{\odot} \mathrm{yr}^{-1}$.

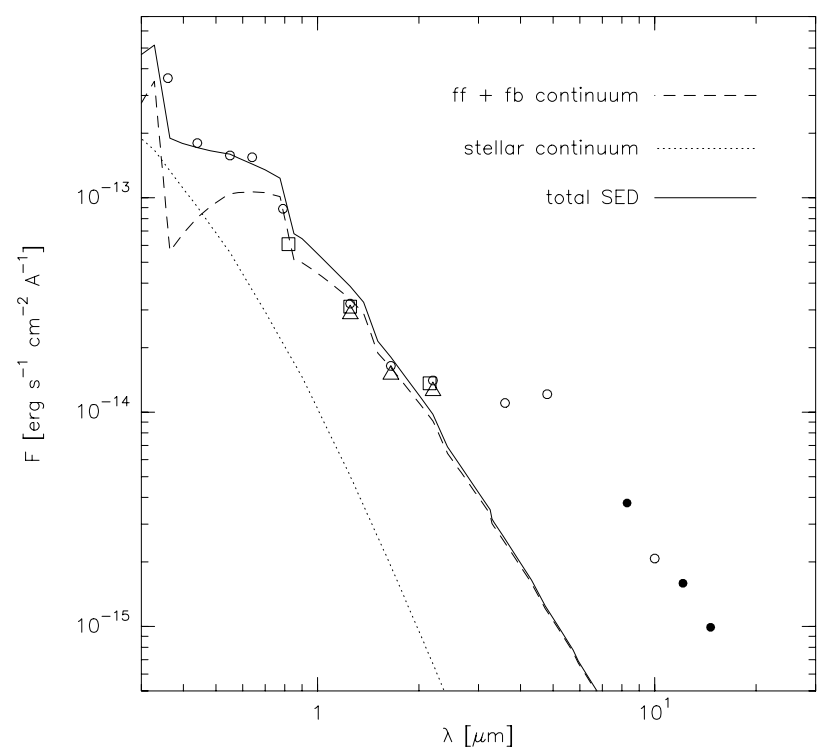

Fig. 8. Calculated SED of R 126 consisting of the stellar continuum and the free-free and free-bound continuum from the polar wind. The theoretical spectrum is reddened to the R 126 value. Photometric data are from Zickgraf et al. (1985, open circles), and from the public databases DENIS (squares), 2MASS (triangles), and MSX (filled circles). Not included here is the modeling of the dust emission to account for the mid-IR excess.

different temperatures. Due to the symmetry and the assumed constant wind temperature, the total observable flux density of the free-free and free-bound emission is given by

$F_{v}=\frac{2 \pi}{d^{2}} \int_{0}^{\zeta_{\max }} B_{v}(T)\left(1-\mathrm{e}^{-\tau(\zeta)}\right) \zeta \mathrm{d} \zeta$

where $\zeta_{\max }$ is the outer edge of the ionized wind and $d$ is the distance to the object. Due to the pole-on view, the dusty disk around R 126 might cause absorption of the free-free and freebound emission from the rear parts of the wind (i.e. below the disk). However, as stated by Kastner et al. (2006), the dust is expected to exist only at large distances, i.e. beyond 120 AU. For the wind densities of our interest, we found that the free-free and free-bound emission is generated much closer to the star, and any absorbing influence of the dust can be neglected.

To calculate the electron density at every point in the wind, we need to know the polar mass loss rate and terminal velocity. Literature values for R 126 are still not well constrained and vary over a huge range, depending on the geometry and the method used by individuals to derive them. As an example serves the terminal velocity for which Bjorkman (1998) gives $650 \mathrm{~km} \mathrm{~s}^{-1}$ while Zickgraf et al. (1985) found $1800 \mathrm{~km} \mathrm{~s}^{-1}$. Due to this uncertainty by almost a factor of 3 we calculate the free-free and free-bound emission by varying the density parameter, i.e. the ratio $F_{\mathrm{m} \text {,pol }} / v_{\infty}$, instead of the individual parameters $F_{\mathrm{m} \text {,pol }}$ and $v_{\infty}$. The total continuum, including the stellar emission, is then reddened with the extinction of R 126, and compared with photometric data from the literature. A good fit to the SED in the optical and near-IR is achieved for $F_{\mathrm{m}, \mathrm{pol}} / v_{\infty} \simeq 2.0 \times 10^{-12} \mathrm{~g} \mathrm{~cm}^{-3}$ and $\beta=0.8$ (see Fig. 8 ).

The density parameters for the equatorial and polar wind as summarized in Table 1 result in a the density contrast on the order of $\rho_{\text {eq }} / \rho_{\text {pole }} \simeq 10$. This value turns out to be a lower limit, because all parameters are chosen such that any change 
Table 1. Model parameters for the polar and equatorial (i.e. the diskforming) winds. Please note that the disk parameters only hold for the [OI] line forming region.

\begin{tabular}{lccc}
\hline \hline Wind & $\begin{array}{c}T_{\mathrm{e}} \\
{[\mathrm{K}]}\end{array}$ & $\begin{array}{c}F_{\mathrm{m}} / v_{\infty} \\
{\left[\mathrm{g} \mathrm{cm}^{-3}\right]}\end{array}$ & $q_{\mathrm{e}}$ \\
\hline Polar & 10000 & $2.0 \times 10^{-12}$ & 1.0 \\
Disk-forming & 8000 & $2.2 \times 10^{-11}$ & $4.0 \times 10^{-4}$ \\
\hline
\end{tabular}

(like (i) a smaller disk opening angle; (ii) the existence of a small ionized inner disk part; (iii) a vertical density distribution within the disk due to a latitude dependence of the parameter $n_{\mathrm{H}}\left(R_{\text {in }}\right)$; (iv) a cut-off in [OI] line luminosities before saturation is reached) will immediately request a higher disk density in order to reproduce the observed [OI] line luminosities.

With a disk density parameter of 10 times the polar one, but a disk ionization fraction of less than $10^{-3}$, the radial electron density distribution within the disk is at least a factor of 100 lower than the one in the polar wind. A contribution of the disk to the free-free and free-bound continuum is therefore indeed negligible, justifying our assumption that the free-free and free-bound emission is generated purely within the polar wind.

\section{Discussion}

For our description of the disk model in Sect. 4.1 we made two severe assumptions, namely we requested that (i) the disk is neutral in hydrogen right from the stellar surface, i.e. $R_{\text {in }} \simeq R_{*}$, and that (ii) the disk has a constant outflow velocity. We will now discuss the validity of these assumptions.

A hydrogen neutral disk right from the stellar surface. We requested that the disk around the rather hot central star is predominantly neutral in hydrogen right from the stellar surface. Recent ionization structure calculations by Kraus \& Lamers (2003) in non-spherical winds of $\mathrm{B}[\mathrm{e}]$ supergiants have shown that in the equatorial region hydrogen can indeed recombine close to the stellar surface, resulting in a geometrically thick hydrogen neutral disk. To quantify this, we refer to their model $\mathrm{C}$ which was calculated for a star with the same effective temperature as $\mathrm{R}$ 126. Further parameters given in their Table 1 were an electron temperature of $10000 \mathrm{~K}$, and an equatorial surface density of $3.3 \times 10^{12} \mathrm{~cm}^{-3}$. With these parameters, Kraus \& Lamers (2003) found that hydrogen recombines in the equatorial plane at a distance of $r \lesssim 1.0004 R_{*}$.

Our model calculations for the [OI] line luminosities revealed a disk density parameter of about $2.2 \times 10^{-11} \mathrm{~g} \mathrm{~cm}^{-3}$ (see Table 1) which results in an equatorial density on the stellar surface of $n_{\mathrm{H}}\left(R_{*}\right) \simeq 10^{13} \mathrm{~cm}^{-3}$. This density is about 3 times higher than the one used by Kraus \& Lamers (2003). Consequently, recombination of the disk material will happen even closer to the stellar surface. It is therefore reasonable to adopt that the disk around the $\mathrm{B}[\mathrm{e}]$ supergiant $\mathrm{R} 126$ is neutral in hydrogen right from the stellar surface.

The constant disk outflow velocity. In order to describe the disk density distribution with only one free parameter, i.e. the density parameter $n_{\mathrm{H}}\left(R_{\text {in }}\right) \sim F_{\text {m,disk }} / v_{\text {out }}=$ const, we set the disk outflow velocity constant within the [OI] line forming region. Figure 5 shows that the line luminosities of the [OI] lines saturate within about $500 R_{*}$; the $5577 \AA$ line saturates even within $50 R_{*}$. Let us assume that the disk-forming wind also has a velocity distribution according to the $\beta$-law. Such a velocity law then mainly influences the formation of the $5577 \AA$ line. This line is created mainly in those wind parts in which the wind is still accelerating, while the other two [OI] lines are unaffected from a $\beta$-type velocity law. Their luminosities are produced at distances at which the velocity has reached already its terminal value.

According to the equation of mass continuity, Eq. (4), a lower velocity at a certain distance results in a higher wind density and therefore in a higher level population. Consequently, the luminosity of the $5577 \AA$ line arising in the accelerated wind region is somewhat enhanced compared to the luminosity created in an outflow of constant (i.e. terminal) velocity. A higher line luminosity, however, results in a lower $6300 \AA / 5577 \AA$ line ratio. Therefore, in order to fit the observations, a slightly different combination of disk ionization fraction and density parameter will be necessary.

We calculated the disk ionization fraction and density parameter for an outflowing disk with a temperature of $8000 \mathrm{~K}$ and a velocity law with $\beta=0.8$. From our fitting procedure we found the following results: $q_{\mathrm{e}} \simeq 10^{-3}$ and $F_{\mathrm{m} \text {,disk }} / v_{\infty} \simeq 1.7 \times$ $10^{-11} \mathrm{~g} \mathrm{~cm}^{-3}$. Even though the ionization fraction is a factor of 2.5 higher than in the case of the constant outflow velocity (see Table 1), the total amount of ionized hydrogen in the disk is still only $0.1 \%$. In addition, the total disk mass loss rate found with the $\beta$-type disk wind scenario is now $\dot{M}_{\text {disk }} \simeq 2 \times$ $10^{-5} M_{\odot} \mathrm{yr}^{-1}$. This value is only slightly lower than the value derived with the constant outflow velocity scenario in Sect. 4.3. Therefore, the conclusion of a hydrogen neutral disk with a density ratio of about a factor of 10 between equatorial and polar wind remains valid.

We want to emphasize that not much is known about the real velocity distribution of the outflowing disks around $\mathrm{B}[\mathrm{e}] \mathrm{su}-$ pergiants. Since a $\beta$-type velocity law does not drastically alter the results, the assumption of a constant outflow velocity as a first guess seems therefore to be a reasonable approach.

\section{Conclusions}

This paper is not aimed to present a detailed analysis of R 126 and to derive its wind and disk parameters with high accuracy, but to present a somewhat revised model for the outflowing disk scenario. We suggest that the disks around $\mathrm{B}[\mathrm{e}]$ supergiants are neutral in hydrogen at, or close to the stellar surface. Indications for such a model come from observations as well as from theory and are here briefly summarized:

- Former investigations of the disks around $\mathrm{B}[\mathrm{e}]$ supergiants assumed that the observed near-IR excess is caused by freefree emission from the disk, as it is the case for classical Be stars. A contribution from the polar wind was thought to be neglibible. Fitting of the near-IR excess with free-free emission arising purely in the outflowing disk delivers a disk mass loss rate that defines an upper limit to the disk (dust) density. The resulting dust emission was however found to be too low to account for the observed IR excess (Porter 2003).

- Porter (2003) tried to solve this dust emission problem by allowing for a flatter disk density distribution. However, his modified disk model for the $\mathrm{B}[\mathrm{e}]$ supergiant $\mathrm{R} 126$ is not able to reproduce the observed [OI] line luminosities, underestimating them by about a factor of 50 ! To account for the [OI] line luminosities a (much) higher disk density is needed.

- Due to the nearly equal ionization potentials of OI and HI, the existence of strong [OI] emission further requests that in the line forming region hydrogen must be predominantly neutral. 
- Recent ionization structure calculations in non-spherically symmetric winds of $\mathrm{B}[\mathrm{e}]$ supergiants have revealed that the wind material indeed recombines in the equatorial plane already at, or very close to the stellar surface, resulting in a hydrogen neutral equatorial disk or zone (see e.g. Kraus \& Lamers 2003, 2006; Kraus 2006).

The most important consequence of such a hydrogen neutral disk is the fact that the disk can no longer be considered as the formation location of the free-free emission causing the near-IR excess. This emission must then mainly be produced in the linedriven polar wind. Consequently, the fitting of the free-free emission does not result in an upper limit to the disk mass loss rate anymore. Instead, the disk mass loss rate, and hence the disk density, can be (much) higher, providing an ideal environment for the [OI] line emission as well as for efficient dust condensation to account for the observed IR excess.

We tested the scenario of a hydrogen neutral disk for the LMC B[e] supergiant R 126 by modeling the line luminosities and line ratios of the [OI] emission lines resolved in our highresolution optical spectra. The parameters derived for the disk and wind of R 126 are the following:

- We found that the [OI] $6300 \AA / 5577 \AA$ line ratio is very sensitive to the ionization fraction in the disk. From fitting the observed line ratio we can conclude that hydrogen in the disk is ionized by less than $0.1 \%$. This confirms that the disk is indeed predominantly neutral in hydrogen.

- The disk mass loss rate of $\dot{M}_{\text {disk }} \gtrsim 2.5 \times 10^{-5} M_{\odot} \mathrm{yr}^{-1}$ found from our fitting is about a factor of 10 higher than the value used by Porter (2003). This is in good agreement with the postulated need for a higher disk density in order to fit the [OI] lines, and also in good agreement with the total dust mass of about $3 \times 10^{-3} M_{\odot}$ as derived by Kastner et al. (2006). A disk with (much) higher density provides a much better environment for efficient dust condensation than the disk model used by Porter (2003).

- The near-IR excess is fitted with free-free and free-bound emission from the B-type line-driven polar wind. The resulting density contrast between equatorial and polar wind is on the order of 10. This value is found to be a lower limit.

To summarize, based on a detailed investigation of the emerging [OI] emission lines we found that the disk around the
$\mathrm{B}[\mathrm{e}]$ supergiant $\mathrm{R} 126$ is neutral in hydrogen right from the stellar surface. Since all B[e] supergiants show strong [OI] line emission, we postulate that the hydrogen neutral ouflowing disk scenario might also hold for the other members of the $\mathrm{B}[\mathrm{e}]$ supergiant class.

Acknowledgements. We thank the anonymous referee for suggestions and critical comments that helped to improve the paper. M.K. acknowledges financial support from GA ČR 205/04/1267. M.B.F. is supported by $C N P q$ (Post-doc position - 150170/2004-1).

\section{References}

Bjorkman, J. E. 1998, in B[e] stars, ed. A. M. Hubert, \& C. Jaschek (Kluwer Academic Publishers), 194

Grevesse, N., \& Sauval, A. J. 1998, Space Sci. Rev., 85, 161

Hamuy, M., Suntzeff, N. B., Heathcote, S. R., et al. 1994, PASP, 106, 566

Kafatos, M., \& Lynch, J. P. 1980, ApJS, 42, 611

Kastner, J. H., Buchanan, C. L., Sargent, B., \& Forrest, W. J. 2006, ApJ, 638, L 29

Kraus, M. 2000, Ph.D. Thesis, University of Bonn, Germany

Kraus, M. 2006, A\&A, 456, 151

Kraus, M., \& Borges Fernandes, M. 2005, in The nature and evolution of disks around hot stars, ed. R. Ignace, \& K. G. Gayley (San Francisco: ASP), ASP Conf. Ser., 337, 254

Kraus, M., \& Lamers, H. J. G. L. M. 2003, A\&A, 405, 165

Kraus, M., \& Lamers, H. J. G. L. M. 2006, in Stars with the B[e] Phenomenon, ed. M. Kraus, \& A. S. Miroshnichenko (San Francisco: ASP), ASP Conf. Ser., 355, 57

Kraus, M., Borges Fernandes, M., Andrade Pilling, D., \& de Araújo, F. X. 2006, in Stars with the B[e] Phenomenon, ed. M. Kraus, \& A. S. Miroshnichenko (San Francisco: ASP), ASP Conf. Ser., 355, 163

Lamers, H. J. G. L. M., \& Cassinelli, J. P. 1999, Introduction to stellar winds (Cambridge University Press)

Lamers, H. J. G. L. M., Zickgraf, F.-J., de Winter, D., Houziaux, L., \& Zorec, J. 1998, A\&A, 340, 117

McGregor, P. J., Hillier, D. J., \& Hyland, A. R. 1988, ApJ, 334, 639

McGregor, P. J., Hyland, A. R., \& McGinn, M. T. 1989, A\&A, 223, 237

Mendoza, C. 1983, IAU Symp., 103, 143

Porter, J. M. 2003, A\&A, 398, 631

Wiese, W. L., Smith, M. W., \& Glennon, B. M. 1966, Atomic Transition Probabilities (National Standard Reference Data System, Washington DC), Vol. 1

Zickgraf, F.-J. 1992, in Nonisotropic and Variable Outflows from Stars, ed. L. Drissen, C. Leitherer, \& A. Nota (San Francisco: ASP), ASP Conf. Ser., 22, 75

Zickgraf, F.-J., Wolf, B., Stahl, \& Humphreys, R. M. 1989, A\&A, 220, 206

Zickgraf, F.-J., Wolf, B., Stahl, O., Leitherer, C., \& Klare, G. 1985, A\&A, 143, 421 